\title{
Long-term outcome of Tunisian children with primary ciliary dyskinesia confirmed by transmission electron microscopy.
}

\author{
Hamouda Samia ${ }^{1}$, Boussetta Khadija ${ }^{1}$, HamzaouiAgnes ${ }^{1}$, Khalsi Fatma ${ }^{1}$, Trabelsi Ines ${ }^{1}$, \\ Jaafoura Hafedh ${ }^{2}$, Tinsa Faten ${ }^{1}$
}

1. Boussetta, Khadija; Bechir Hamza Children\'s Hospital of Tunis, Department B

2. Jaafoura, Hafedh; Laboratory of Histology of Medecine University of Tunis -Tunis -Tunisia

\begin{abstract}
Background: Primary ciliary dyskinesia (PCD) is rare. Its diagnosis requires experienced specialists and expensive infrastructure. Its prognosis is variable.

Objective: To study the long-term outcome of PCD in Tunisian children with ciliary ultra-structure defects detected by electron microscope.

Methods: Covering a period of 20 years (1996-2015), this retrospective study included all patients with definite PCD (outer dynein arms (DA) defects and/or situs inversus) and presumed PCD (other ciliary ultra-structure defects). The clinical data and the investigations made were registered at diagnosis and during the follow-up.

Results: Patients with a definite PCD $(\mathrm{G} 1, \mathrm{n}=7)$ were diagnosed earlier compared to those with a presumed PCD (G2, $n=13)$ (2.5 vs. 9.3 years on average). At diagnosis, bronchiectasis was more frequent in G1 (3/7 vs. 4/13). The inner DA loss was constant in G1 and predominant in G2. The treatment adhesion was more often irregular in G2 (2/7 vs. 8/13). During a mean follow-up of 11 years, G1 showed less severe outcome (clubbing (0 vs. 3), bronchiectasis (3 vs. 11; more expanded in G2), proximal and distal airway obstruction (0/3 vs. 5/7), lobectomy (0 vs. 2$)$, and death (0 vs. 2)).
\end{abstract}

Conclusion: Precocious diagnosis and regular treatment may enhance the PCD prognosis.

Keywords: Primary ciliary dyskinesia, cilia, child, recurrent pneumonia, bronchiectasis.

DOI: http://dx.doi.org/10.4314/ahs.v16i4.11

Cite as: Samia H, Khadija B, Agnès H, Fatma K, Ines T, Hafedh J, Faten T. Long-term outcome of Tunisian cbildren with primary ciliary dyskinesia confirmed by transmission electron microscopy. Afri Health Sci 2016;16(4): 954-961. bttp:/ / dx.doi.org/10.4314/ahs.v16i4.11

\section{Introduction}

Primary ciliary dyskinesia (PCD) is a rare inherited autosomal-recessive disease. In Europe, the prevalence of PCD is estimated at $1 / 10000$. It is higher in certain regions, especially in North Africa probably because of a high rate of consanguinity ${ }^{1,2}$. PCD is caused by an abnormal structure and/or function of the epithelial cell cilia and sperm tails. In the respiratory system, this anomalyimpairs mucociliary clearance and leads to recurrent infections of the upper and lower airways in the first months of life ${ }^{3}$.

The PCD diagnosis requires an expensive infrastructure and an experienced team of clinicians and scientists. In

\section{Corresponding author: \\ Hamouda Samia, \\ Boussetta, Khadija; Bechir Hamza Children\'s \\ Hospital of Tunis, Department B \\ Email: samia.hamouda@gmail.com}

children, other chronic respiratory diseases, such as cystic fibrosis (CF), are often investigated before starting appropriate and complex PCD examination. The electron microscopic cilia study from a nasal or bronchial sample can detect the cilia defects, mainly in the dynein arms (DA) from the outer doublets, or a missing central pair associated with a disarrangement of the microtubules. The ciliary beat frequency can be assessed by a light microscopy and more recently by a high speed video-microscopy, available only in specialist centers across the globe ${ }^{1,4}$. The PCD medical treatment, based on daily airway clearance and antibiotics, is not standardized. It is usually extrapolated from $\mathrm{CF}$ and non-CF bronchiectasis literature. The PCD monitoring should include regular sputum examination and lung function tests. The prognosis is variable. However, it is known to be better than the CF one $e^{5,6}$.

In Tunisia, PCD is under-diagnosed. It needs a specialist physician to be recognized. The definitive diagnosis is difficult to establish. The unique tool to support PCD diagnosis at present is the electron microscope with a African Health Sciences Vol 16 Issue 4, December, 2016 
limited availability. This microscope cannot detect all the PCD cases in Tunisia since patients with normal ciliary ultra-structure can have a genetically proven PCD7. On the other hand, for some ciliary abnormalities (an outer DA defect and a combined inner and outer DA defect), the PCD diagnosis is definitive. For others (an inner DA defect alone, or combined with a radial spoke defect), repeating the test to avoid the possibility of a false-positive diagnosis is recommended ${ }^{8}$. This procedure cannot be fulfilled in our context given its cost. Because of these obstacles, the specialist most often retains the PCD diagnosis only on clinical and radiological bases and after having excluded other major chronic respiratory diseases. In addition, confirming PCD diagnosis is not the only challenge. In fact, the management of PCD in children requires a specialized paediatrician. The monitoring depends closely on the availability of access to medical health care. The aim of this study was to assess the outcome of this rare disease in Tunisian children with detected ciliary ultra-structure defects.

\section{Methods}

\section{Study design}

This retrospective study was carried out in child and adult pulmonology referral units (Children's Department B in Bechir Hamza Children's Hospital of Tunis and Department $C$ of Abderrahman Mammi Hospital of Ariana, respectively) from 1996 to 2015. We collected all patients with a definitive diagnosis of PCD based upon ciliary ultra-structure defects. In all cases, CF and immunodeficiency were excluded by appropriate examinations (sweat test, immune investigation). The ciliary ultra-structure defects were identified by an electron microscope cilia study, performed from nasal biopsy, ciliary brushing and/or bronchial biopsy. At least 100 ciliary cross-sections from healthy areas of the respiratory epithelium per patient were analyzed. The PCD diagnosis was established when more than $40 \%$ of the cilia were damaged. The patients regarded as affected with PCD because of a compatible clinical and radiological phenotype without any ciliary ultra-structure abnormalities or without an electron microscopic cilia study were not included in this study.

Two groups were determined. Group 1 corresponding to a definite PCD and included patients with outer DA defects and/or situs abnormalities. Group 2 related to a presumed PCD and included the patients with other ciliary ultra-structure defects and without situs inversus.

\section{Data collection}

Data was collected from patients' medical records. For each patient, we noted the age at first symptoms and at diagnosis and the circumstances of the PCD discovery. We registered the existence of a family history of PCD. We analysed the clinical data at diagnosis and during the monitoring as well as the results of the different investigations made. The clinical data specified the presence of recurrent or persistent upper and lower respiratory symptoms. The radiological exploration included a chest x-ray, x-ray of the sinuses, chest CT scan, and echocardiography. When a situs inversus was found on the chest x-ray, an abdominal ultrasound was performed to verify the mirror image arrangement of the internal organs. The sputum culture was carried out at diagnosis, during an acute respiratory exacerbation and/or during the monitoring. The lung function tests were performed by plethysmography. They were based on the measurement of the specific airway resistance for the children aged more than four and on spirometry for those aged more than seven. The hearing acuity was assessed by audiometry during the monitoring. The management of the disease was also analyzed. The minimal required duration of the follow-up was one year.

\section{Statistical analysis}

This study was cross-sectional and descriptive. The data were entered using Excel software as qualitative and quantitative values. The variables were expressed as percentages and compared using the Chi square test. The Chi-square test associations were appropriate. The significance level of " $p$ " was 0.05 .

\section{Results}

Twenty patients were registered as having PCD with ciliary ultra-structure defects. Group 1 (definite PCD) and group 2 (presumed PCD) included seven and 13 patients respectively. In group 1 , the patients were divided into four boys and three girls. The average age at diagnosis was 2.5 years extremes: 3 months; 12 years. Four patients had a complete situs inversus relevant to Kartagener'ssyndrome. The diagnosis of their PCD was earlier at the mean age of 6.5 months extremes: 3; 12 months (Table 1).

Parental consanguinity was found in four cases. The analysis of the family history showed symptoms suggestive of PCD (recurrent respiratory infections, infertility) in three cases. 
Table 1: Patients' age at diagnosis and the primary ciliary dyskinesia diagnosis delay

\begin{tabular}{cccccc}
\hline $\begin{array}{c}\text { Patients from group } \\
\text { with situs inversus }\end{array}$ & $\begin{array}{c}\text { Patients from } \\
\text { group } 1 \\
\text { without situs } \\
\text { inversus }\end{array}$ & $\begin{array}{c}\text { Total population of } \\
\text { group } 1\end{array}$ & $\begin{array}{c}\text { Patients from group } \\
\text { Total population of } \\
\text { the study }\end{array}$ \\
Average $\quad$ Range & Average $\quad$ Range & Average & Range & Average $\quad$ Range Average $\quad$ Range \\
\hline
\end{tabular}

\begin{tabular}{|c|c|c|c|c|c|c|c|c|c|c|}
\hline $\begin{array}{l}\text { Age at } \\
\text { diagnosi } \\
\text { s }\end{array}$ & $\begin{array}{c}6.5 \text { month } \\
\mathrm{s}\end{array}$ & $\begin{array}{c}3- \\
12 \mathrm{month} \\
\mathrm{s}\end{array}$ & 5 years & $\begin{array}{c}8 \\
\text { months- } \\
12 \text { years }\end{array}$ & 2.5 years & $\begin{array}{c}3 \\
\text { months - } \\
12 \text { years }\end{array}$ & 9.3 years & $\begin{array}{c}18 \\
\text { months - } \\
13 \text { years }\end{array}$ & 6.9 years & $\begin{array}{c}3 \\
\text { months- } \\
13 \text { years }\end{array}$ \\
\hline
\end{tabular}
Time
fordias
1 -
8
nonths-
2.2 years
1 month -
6.3 years
months -
11 years

The PCD was revealed by recurrent pneumonia in four cases, persistent wheezing in two cases, and chronic cough in one case. The neonatal respiratory distress was retrospectively found in three cases, always taken for a maternal-fetal infection. At diagnosis, the physical examination showed wheezing $(n=6)$ and crackles $(n=4)$. In the ear, nose and throat (ENT) examination, three patients had upper respiratory symptoms including purulent rhinitis, sinusitis and/or chronic otitis. One child aged 12 had nasal polyposis. The radiological abnormalities were mainly situs inversus $(n=4)$ and lung distension $(n=4)$ (Figure 1). The middle lobe was as much affected as the right upper lobe ( $\mathrm{n}=2$ for each lobe). The patient aged $12 \mathrm{had}$ normal lung function tests. The other patients hadn't had these tests because of their age.

\section{Figure 1: Chest Radiological data at diagnosis}

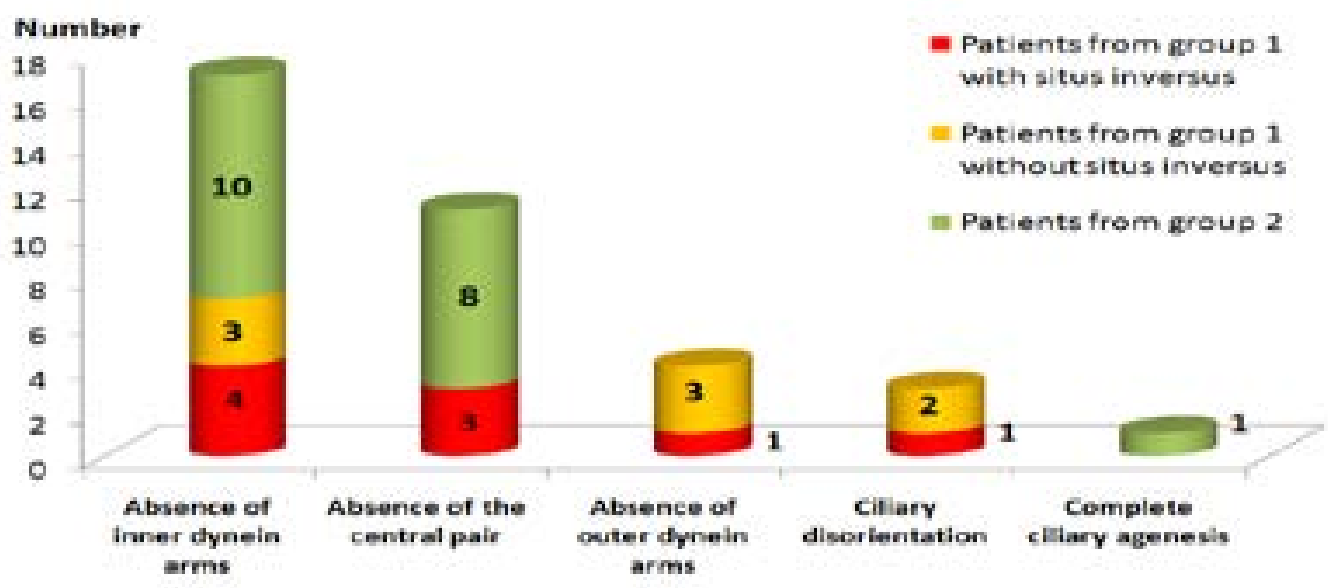

Compared to group 1, most patients from group 2 were older at diagnosis and the diagnosis delay was about three times longer (Table 1). Their clinical characteristics are illustrated in table 2 .

The dominant chest imaging abnormalities were lung distension and localized or diffuse alveolaro pacities (Figure 1). The middle lobe was affected in most of the cases $(n=10)$. The sinus radiography showed a unilateral $(n=1 / 8)$ or bilateral maxillary sinusitis $(n=7 / 8)$. The pulmonary function tests showed airway obstruction in most cases $(n=6 / 7)($ Table 3$)$. 
Table 2: Clinical characteristics at diagnosis of group 2

\begin{tabular}{|c|c|}
\hline Clinical features of group 2 & Number of cases \\
\hline Gender : $M$ / F & $10 / 3$ \\
\hline Parental consanguinity & 6 \\
\hline Family history suggestive of primary ciliary dyskinesia & 2 \\
\hline Respiratory distress at birth & $\mathbf{1}$ \\
\hline Circumstances of $P C D$ discovery: & 13 \\
\hline - recurrent lower respiratory infections & 5 \\
\hline - persistent wheezing & 5 \\
\hline - chronic cough & 2 \\
\hline - chronic respiratory failure & 1 \\
\hline Physical examination findings at diagnosis: & 13 \\
\hline - wheezing & 8 \\
\hline - crackles & $\begin{array}{l}8 \\
2\end{array}$ \\
\hline - clubbing & 1 \\
\hline - nasal polyposis & 1 \\
\hline $\begin{array}{l}\text { - other upper respiratory symptoms (purulent rhinitis, } \\
\text { sinusitis and/or chronic otitis) }\end{array}$ & 7 \\
\hline
\end{tabular}

Table 3: Lung function test data at diagnosis of group 2

\begin{tabular}{lcc}
\multicolumn{1}{c}{ Lung function tests data } & $\begin{array}{c}\text { Number of } \\
\text { patients }\end{array}$ & Values \\
\hline - Normal & $\mathbf{1}$ & \\
- Proximal airway obstruction: & $\mathbf{2}$ & $180-160 / 152-120$ \\
- Pre-/ Post $\beta 2$-agonist sRaw \% pred & & $69-67 / 71-77$ \\
- Pre-/ Post $\beta 2$-agonist FEV1 \% pred & $\mathbf{3}$ & $24-38-44 / 33-45-48$ \\
- Distal airway obstruction: & & \\
- Pre-/ Post $\beta 2$-agonist FEF25-75\% \% pred & $\mathbf{1}$ & $450 \mathrm{ml}(22 \%)$ \\
- Major airway obstruction associated with a & & $1020 / 805$ \\
\hline
\end{tabular}

SRaw: specific airway resistance; FEV1: forced expiratory volume at one second; FEF2575\%:forced expiratory flow between $25 \%$ and $75 \%$ of forced vital capacity; \%pred: $\%$ of predicted value.

The ultra-structural cilia study was performed from a nasal biopsy in seven cases, and a ciliary brushing and bronchial biopsy in 13 cases. The ciliary defect affected the majority of the cell cilia in all cases. The main defect was the absence of the inner DA ( $\mathrm{n}=17)$. It was constant in group 1, and isolated in four cases in group 2 (Figure 2 and 3).

Figure 2: Ultra-structural ciliary abnormalities according to the electron microscopic study

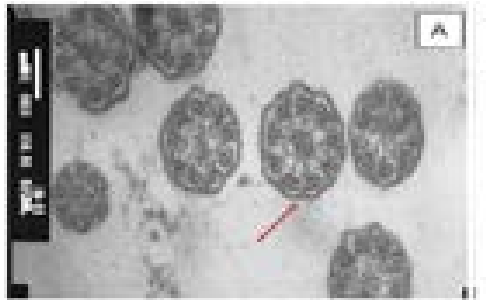

In group 2, in the absence of outer DA defects and situs inversus, the PCD diagnosis was retained given the clinical data (consanguinity, family history of infertility, and/ or association of upper and lower chronic respiratory symptoms) and the investigation results (preferential involvement of the middle lobe on chest imaging, non-reversible airway obstruction on lung function tests, ciliary

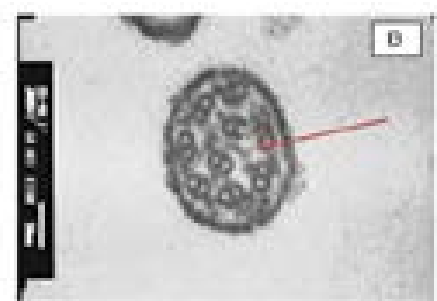

ultra-structure defects in the majority of the cell cilia on the electron microscope, normal sweat test and immune investigation).

The washing of nasal cavities, using isotonic saline, and chest physiotherapy were routinely recommended in all cases. The adhesion to physiotherapy was irregular in half 
the cases (group 1, n=2; group 2, $n=8$ ) owing to financial difficulties and the remoteness of the physiotherapy centre. All patients received antibiotics during an acute respiratory tract infection. A rotating antibiotic therapy was indicated for eight children (group 1, $n=4$; group 2, $\mathrm{n}=4$ ) in the cold season because of their young age, a diffuse bronchiectasis, and/or difficult access to healthcare. It alternated the amoxicillin/clavulanic acid with the cotrimoxazole. Inhaled corticosteroids were added in half the cases (group 1, $n=2$; group 2, n=8) because of frequent wheezing.

The patients from group 1 had been monitored for 11 years on average extremes: 5; 20 . Five had regular clinical controls every three to six months and two consulted irregularly because of bad financial conditions. All of them had normal growth and no limitation to any physical effort. In the absence of a regular treatment $(n=2)$, patients complained mainly of chronic cough and nasal obstruction. For compliant patients $(n=5)$, symptoms were more attenuated. Bronchiectasis secondarily appeared in three cases. It affected lower lobes as well as the middle lobe. After five years of a regular monitoring, one case of PCD, diagnosed at the age of five months in a compliant patient, has not progressed to bronchiectasis. The respiratory infections occurred one to three times per year, managed as outpatients, and mainly caused by Haemophilus influenzae. The other isolated germs were Moraxella catarrhalis, Streptococcus pneumoniae, Haemophilus parainfluenzae, andKlebsiella pneumoniae. No patient was affected with Pseudomonas aeruginosa. The follow-up of the lung function tests, performed in three cases, showed lung inflation and a non-reversible decrease of the distal airflow with forced expiratory flow between $25 \%$ and $75 \%$ of forced vital capacity (FEF25-75) less than $50 \%$ of predicted value in all cases.

In group 2, two patients had died of acute respiratory distress syndrome (ARDS) after one and four years of follow up (respectively at the age of 13 and 6 (years)). The first one had a severe chronic respiratory failure at diagnosis associated with denutrition, wheezing, clubbing, oxygen dependency, diffuse bronchiectasis and a major airway obstruction associated with a severe restrictive syndrome on the lung function tests (Table 3). The second had a complete ciliary agenesis at the microscopic study. He was eutrophic and had permanent wheezing despite a regular and optimal treatment. His chest CTscan showed important lung distension associated with bilateral bronchiectasis. No germ was found during the fatal respiratory infection in both cases.

The other 11 patients from group 2 had been monitored also for 11 years on average extremes: 5; 14. Those having irregular physiotherapy $(\mathrm{n}=8)$ showed more severe exacerbations. A beginning of clubbing was noticed in three cases. All patients from this group ended up with expanded bronchiectasis. During the respiratory exacerbations, the same bacteria found in group 1 were isolated. Two patients underwent resection of the middle lobe, which was a frequent source of suppuration. In addition to lung inflation and distal airflow decreasing, the lung function tests showed forced expiratory volume at one second (FEV1) less than $80 \%$ of predicted value in five cases out of seven.

For both groups, no persistent pulmonary hypertension was found in echocardiography monitoring. On the other hand, two patients (one from each group) had sensorineural deafness requiring hearing equipment and one patient from group 2 had a nasal polypectomy. Two patients(one from each group) developed mite induced allergic asthma at the age of five and six contributing to in increasing respiratory exacerbations. The respiratory symptoms and the lung function tests improved after long-acting beta agonist inhaled corticosteroids were added.

\section{Discussion}

To our knowledge, this study is the first Tunisian pediatric cohort grouping PCD cases confirmed by histological ciliary study although there are difficulties in ascertaining the PCD diagnosis in our context. The cohort mainly highlighted the fact that despite an important diagnosisdelay (4.8 years on average), the outcome of this chronic disease over a mean period of 11 years extremes: 5; 20 wasn't severe in the majority of cases. The 13-year-old child, who died one year after the PCD diagnosis, was in fact referred to us at an advanced stage of the disease (chronic respiratory failure). Moreover, the cilia ultra-structural abnormalities, detected by a sophisticated technique, allowed the distinction between two groups: definite PCD (group 1) and presumed PCD (group 2). The patients in group 1 were diagnosed earlier and had less severe outcome. In group 2, a child with complete cilia agenesis was always wheezing and died at the age of six. However, the clinical and histological phenotype variations could not explain the outcome difference since the patients of group 2 were less compliant. Because of an 
early diagnosis in group 1, the management of the disease including parental education was precocious probably influencing the treatment compliance. In all cases, the PCD management remained dependent on the socio-economic status of our patients and their ability to have access to healthcare. On the other hand, regular treatment made patients less symptomatic without stopping the progression of the disease, especially for bronchiectasis.

Although the PCD symptoms appear usually early, its diagnosis is delayed with a mean age at diagnosis around five years in developed countries ${ }^{9-11}$. The presence of a situs inversus allows precocious suspicion of $\mathrm{PCD}^{12}$, as in four of our patients. In fact, their PCD diagnosis was established at a mean age of 6.5 months whereas the average age at diagnosis of all patients was 6.9 years. The diagnosis delay is explained by the non-specific respiratory symptoms, first suggesting more frequent childhood conditions, and by the specialized PCD diagnostic tests available only in distinguished institutions. Indeed, most patients had several examinations (for example: sweat test, immune investigation, allergic tests) before the PCD diagnosis. The situs inversus is known to be present in around $50 \%$ of PCD cases. It is one element of the Kartagener's syndrome triad. It is explained by a random distribution happening in the absence of the leftward side flow induced by normal embryonic cilia rotation ${ }^{12,13}$. In our series, it was observed in $4 / 7$ cases of the definite PCD (group 1). Nevertheless, it was reported in $20 \%$ of all the cases because most physicians take for granted the diagnosis of Kartagener's syndrome in children with situs inversus and upper and lower respiratory infections without making a transmission electronic microscopy study. These patients were not included in this study.

The clinical feature of the PCD is often homogeneous combining upper and lower respiratorymanifestations. The respiratory symptoms are present in $50-90 \%$ of cases at birth consisting of a transient tachypnea or ventilatory disorder ${ }^{14,15}$. Their favorable evolution delays the diagnostic step. A recent case-control study identified in term neonates with unexplained respiratory distress three factors to be considered by clinicians to better detect PCD: lobar collapse, situs inversus, and/or more than two day-long oxygen therapy ${ }^{16}$. In childhood, the chronic wet cough, persistent wheezing, recurrent pneumonia and bronchiectasis lead to diagnosis when current explorations return negative. The ENT manifestations are early and can be in the foreground. In this study, half the patients had per- manent ENT symptoms at diagnosis, less than in the series of Beucher et $\mathrm{al}^{9}$ and Sommer et $\mathrm{al}^{17}$ (86\% and 81\% respectively). The high prevalence of the ENT morbidity in PCD should familiarize ENT specialists themselves with the disease to investigate patients in time.

The measurement of nasal nitric oxide (NO) is a good screening and non-invasive test in older children. The PCD is strongly suspected when the rate of nasal NO is lowered ${ }^{18}$. This measure wasn't available in our country during the study period. However, because it is not reliable in preschool children, this technique has a limited usefulness for early diagnosis.

The analysis of ciliary ultra-structure using transmission electron microscopy leads to PCD confirmation in most of the cases $^{14}$. This method requires time, expansive equipment and experienced specialists for sampling and specimen analysis. The impairment of DA was more frequent than the absence of the central pair in this study as well as in different series ${ }^{19,20}$.

The correspondence between genetic mutation and cytological abnormalities was demonstrated, while the link between genetic analysis and clinical phenotype was more difficult to establish 3,21,22. A French retrospective study conducted among 60 children with PCD highlighted the relationship between the severity of clinical presentation and the ultra-structural ciliary defect. The respiratory symptoms of patients with DA loss occurred earlier and were less severe at diagnosis compared to patients with central pair abnormalities. The outcome was worse in the first group ${ }^{23}$. In this study, in group 1 which included the absence of inner and outer DA, PCD was diagnosed earlier but the patients had more bronchiectasies at diagnosis than in group 2 which included the absence of central pair (3/7 vs. $4 / 13)$. The adherence to treatment influenced the PCD outcome in both groups. The PCD genetic analysis was implemented in our country in 2015. Its results are ongoing.

The PCD induces severe respiratory impairment but remains accessible to medical treatment (chest physiotherapy and antibiotics). The treatment must be early and regular. The ultimate solution for a completely destroyed and infected lobe is resection ${ }^{20}$. The Haemophilus influenzae is the most isolated germ during lung infections ${ }^{5,6}$, as in this study. It was confirmed also in a recent European 
multicenter study enrolling 158 children with PDC. This pathogen was found at least once in $65 \%$ of the patients. Pseudomonas aeruginosa was isolated in 58 patients (37\%) of whom eight $(5 \%)$ were chronically infected. It wasn't associated with spirometry changes unlike in $\mathrm{CF}^{24}$. No sputum culture showed this bacterium in our patients.

Maglione et $\mathrm{al}^{25}$ compared the change over time of the lung structure in Chest CT-scan to the lung function in spirometry in 20 children with PCD. They concluded that CT scan scores deteriorated with age, while spirometry did not change significantly, or might even have improved, suggesting that spirometry was less sensitive in assessing lung disease progression in PCD. Moreover, there was no correlation between the changes in CT scan scores and in spirometry.

Chronic rhinosinusitis significantly impacts children's quality of life in PCD. The surgery indications include nasal obstruction and polyposis. Endoscopic sinus surgery and debridement of sinonasal polyps are frequently performed with variable results ${ }^{26}$.

\section{Conclusion}

PCD remains under-recognized by physicians. A better awareness of the clinical features of the disease may help induce an early diagnosis and a precocious management. A regular treatment could help reduce disease morbidity.

\section{Limitations of the study}

It should be pointed out nevertheless that the limitations of this study with regard to the diagnosis of group 2 were imposed mainly by the absence of a PCD genetics study and ciliary functional tests carried out by high-speed video-microscopy in Tunisia.

\section{Source of Support:}

Nil

\section{Conflict of interest:}

None declared.

\section{References}

1. Lucas JS, Burgess A, Mitchison HM, Moya E, Williamson M, Hogg C. Diagnosis and management of primary ciliary dyskinesia. Arch Dis Child. 2014;99:850-6. PubMed 2. Verra F, Escudier E, Bignon J, et al. Inherited factors in diffuse bronchiectasis in the adult: a prospective study. Eur. Respir. J. 1991;4:937-44.
3. Stillwell PC, Wartchow EP, Sagel SD. Primary ciliary dyskinesia in children: A review for pediatricians, allergists, and pediatric pulmonologists. Pediatr Allergy Immunol Pulmonol.2011;24:191-196.

4. Lucas JS, Leigh MW. Diagnosis of primary ciliary dyskinesia: searching for a gold standard. Eur Respir J. 2014;44:1418-22.

5. Deepika P, Stephanie DD, Sharon DD. Treatment recommendations in primary ciliary dyskinesia. Paediatr Respir Rev. 2016;18:39-45 PubMed.

6. Sagel SD, Davis SD, Campisi P, Dell SD. Update of respiratory tract disease in children with primary ciliary dyskinesia. Proc Am Thorac Soc. 2011;8:438-43 PubMed .

7. Knowles MR, Leigh MW, Carson JL, et al. Mutations of DNAH11 in primary ciliary dyskinesia patients with normal ciliary ultrastructure. Thorax. 2012; 67: 433-41. PubMed

8. O'Callaghan C, Rutman A, Williams GM, Hirst RA. Inner dynein arm defects causing primary ciliary dyskinesia: repeat testing required. Eur Respir J. 2011; 38: 603-607.

9. Beucher J, Chambellan A, Segalen J, Deneuville E. Primary ciliary dyskinesia: a retrospective review of clinical and paraclinical data. Rev Mal Respir. 2011;28:856-63 PubMed.

10. Kuehni CE, Frischer T, Strippoli MF, et al. Factors influencing age at diagnosis of primary ciliary dyskinesia in European children. Eur Respir J. 2010;36:1248-58 PubMed.

11. Hosie PH, Fitzgerald DA, Jaffe A, Birman CS, Rutland J, Morgan LC. Presentation of primary ciliary dyskinesia in children: 30 years' experience. J Paediatr Child Health. 2015;51:722-6.

12. Ciancio N, de Santi MM, Campisi R, Amato L, Di Martino G, Di Maria G. Kartagener's syndrome: review of a case series. Multidiscip Respir Med. 2015;30:10-18.

13. Pennekamp P, Menchen T, Dworniczak B, Hamada H. Situs inversus and ciliary abnormalities: 20 years later, what is the connection? Cilia. 2015;4:1-12.

14. Barbato A, Frischer T, Kuehni CE, et al. Primary ciliary dyskinesia: a consensus statement on diagnostic and treatment approaches in children. Eur Respir J. 2009;34: 1264-76. PubMed

15. Yasuhara J, Yamada Y, Hara K, et al. Primary ciliary dyskinesia diagnosed on nasal mucosal biopsy in two newborns. Pediatr Int. 2014;56:258-61 PubMed.

16. Mullowney T, Manson D, Kim R, Stephens D, Shah V, Dell S. Primary ciliary dyskinesia and neonatal respiratory distress. Pediatrics. 2014;134:1160-6.

17. Sommer JU, Schäfer K, Omran H, et al. ENT maniAfrican Health Sciences Vol 16 Issue 4, December, 2016 
festations in patients with primary ciliary dyskinesia: prevalence and significance of otorhinolaryngologic co-morbidities. Eur Arch Otorhinolaryngol. 2011;268:383-388. 18. Bush A, Chodhari R, Collins N, et al. Primary ciliary dyskinesia: current state of the art. Arch Dis Child. 2007;92:1136-1140. PubMed

19. Plesec T.P, Ruiz A., McMahon JT, Prayson RA. U1trastructural abnormalities of respiratory cilia: a 25 -year experience. Arch. Pathol. Lab. Med. 2008;132:1786-91. PubMed

20. Theegarten D, Ebsen M. Ultrastructural pathology of primary ciliary dyskinesia: report about 125 cases in Germany. Diagn Pathol. 2011;24:115.

21. Davis SD, Ferkol TW, Rosenfeld M, et al. Clinical features of childhood primary ciliary dyskinesia by genotype and ultrastructural phenotype. Am J Respir Crit Care Med. 2015;191:316-24.

22. Imtiaz F, Allam R, , Al-Sayed M. Variation in DNAH1 may contribute to primary ciliary dyskinesia. BMC Med Genet. 2015;17:16-14.

23. Vallet C, Escudier E, Roudot-Thoraval F, et al. Primary ciliary dyskinesia presentation in 60 children according to ciliary ultrastructure. Eur J Pediatr. 2013;172:1053-60. PubMed

24. Maglione M, Bush A, Nielsen KG, et al. Multicenter analysis of body mass index, lung function, and sputum microbiology in primary ciliary dyskinesia. Pediatr Pulmonol. 2014;49:1243-50.

25. Maglione M, Bush A, Montella S, Mollica C, Manna A, Esposito A et al. Progression of lung disease in primary ciliary dyskinesia: is spirometry less accurate than CT? Pediatr Pulmonol. 2012;47:498-504 PubMed.

26. Mener DJ, Lin SY, Ishman SL, Boss EF. Treatment and outcomes of chronic rhinosinusitis in children with primary ciliary dyskinesia: where is the evidence? A quantitative systematic review. Int Forum Allergy Rbinol. 2013;3:986-91 PubMed. 